\title{
RelTextRank: An Open Source Framework for Building Relational Syntactic-Semantic Text Pair Representations
}

\author{
Kateryna Tymoshenko ${ }^{\dagger}$ and Alessandro Moschitti and Massimo Nicosia ${ }^{\dagger}$ and Aliaksei Severyn ${ }^{\dagger}$ \\ ${ }^{\dagger}$ DISI, University of Trento, 38123 Povo (TN), Italy \\ Qatar Computing Research Institute, HBKU, 34110, Doha, Qatar \\ \{kateryna.tymoshenko, massimo.nicosia\} @unitn.it \\ \{amoschitti, aseveryn\}@gmail.com
}

\begin{abstract}
We present a highly-flexible UIMA-based pipeline for developing structural kernelbased systems for relational learning from text, i.e., for generating training and test data for ranking, classifying short text pairs or measuring similarity between pieces of text. For example, the proposed pipeline can represent an input question and answer sentence pairs as syntacticsemantic structures, enriching them with relational information, e.g., links between question class, focus and named entities, and serializes them as training and test files for the tree kernel-based reranking framework. The pipeline generates a number of dependency and shallow chunkbased representations shown to achieve competitive results in previous work. It also enables easy evaluation of the models thanks to cross-validation facilities.
\end{abstract}

\section{Introduction}

A number of recent works (Severyn et al., 2013; Tymoshenko et al., 2016b,a; Tymoshenko and Moschitti, 2015) show that tree kernel methods produce state-of-the-art results in many different relational tasks, e.g., Textual Entailment Recognition, Paraphrasing, question, answer and comment ranking, when applied to syntacticosemantic representations of the text pairs.

In this paper, we describe RelTextRank, a flexible Java pipeline for converting pairs of raw texts into structured representations and enriching them with semantic information about the relations between the two pieces of text (e.g., lexical exact match). The pipeline is based on the Apache UIMA technology ${ }^{1}$, which allows for the creation

\footnotetext{
1https://uima.apache.org/
}

of highly modular applications and analysis of large volumes of unstructured information.

RelTextRank is an open-source tool available at https://github.com/iKernels/ RelTextRank. It contains a number of generators for shallow and dependency-based structural representations, UIMA wrappers for multi-purpose linguistic annotators, e.g., Stanford CoreNLP (Manning et al., 2014), question classification and question focus detection modules, and a number of similarity feature vector extractors. It allows for: (i) setting experiments with the new structures, also introducing new types of relational links; (ii) generating training and test data both for kernel-based classification and reranking, also in a cross-validation setting; and (iii) generating predictions using a pre-trained classifier.

In the remainder of the paper, we describe the structures that can be generated by the system (Sec 2), the overall RelTextRank architecture (Sec 3$)$ and the specific implementation of its components (Sec 4). Then, we provide some examples of how to run the pipeline from the command line $(\operatorname{Sec} 5)^{2}$. Finally, in Sec 6, we report some results using earlier versions of RelTextRank.

\section{Background}

Recent work in text pair reranking and classification, e.g., answer sentence selection (AS) and community question answering (cQA), has studied a number of structures for representing text pairs along with their relational links, which provide competitive results when used in a standalone system and the state of the art when combined with feature vectors (Severyn et al., 2013; Tymoshenko et al., 2016b; Tymoshenko and Moschitti, 2015) and embeddings learned by the neural networks (Tymoshenko et al., 2016a). In this sec-

\footnotetext{
${ }^{2}$ The detailed documentation is available on the related GitHub project.
} 


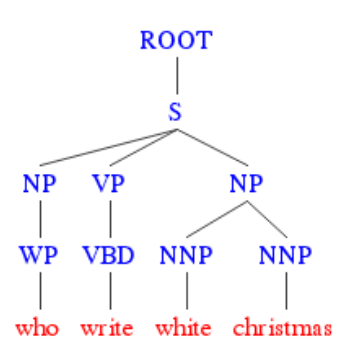

(a) $\mathrm{CH}$

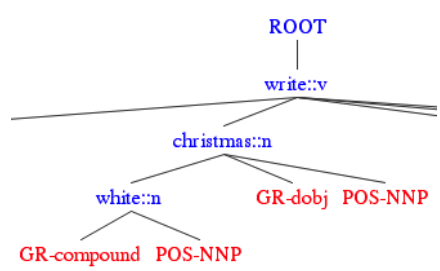

(d) LCT fragment

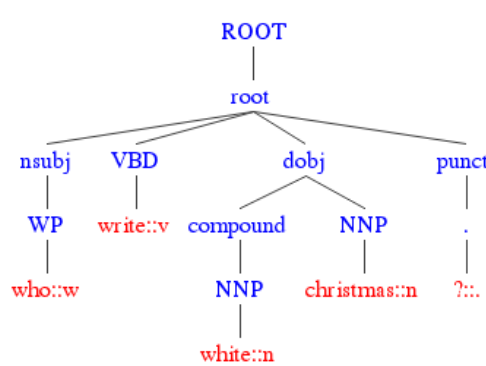

(b) DT1

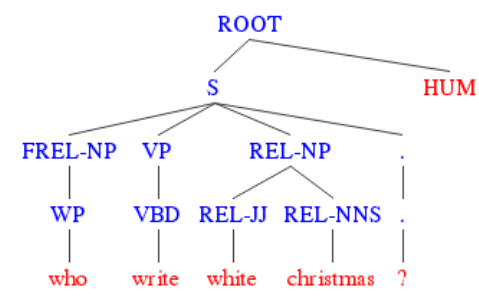

(e) $\mathrm{CH}^{p}$ structure enriched with the relational labels

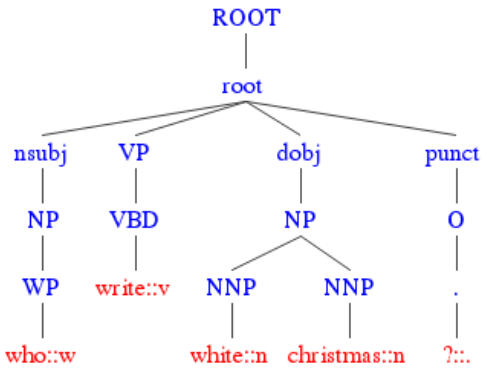

(c) DT2

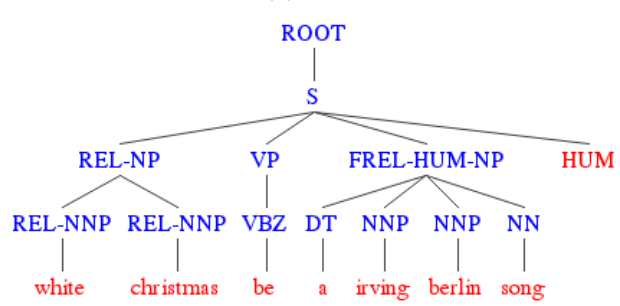

white christmas be a irving berlin song

Figure 1: Structures generated by RelTextRank

tion, we provide an overview of the structures and relational links that can be generated by RelTextRank (specific details are in the above papers).

\subsection{RelTextRank Structures}

RelTextRank can generate the following structural representations for a text pair $\left\langle T_{1}, T_{2}\right\rangle$, where, e.g., $T_{1}$ can be a question and $T_{2}$ a candidate answer passage for an AS task.

Shallow pos-chunk tree representation $(\mathrm{CH}$ and $\mathbf{C H}^{p}$ ). Here, $T_{1}$ and $T_{2}$ are both represented as shallow tree structures with lemmas as leaves, their POS-tags as their parent nodes. The POStag nodes are further grouped under chunk and sentence nodes. We provide two versions of this structure: $\mathrm{CH}^{p}$ (Fig. 1e), which keeps all information while $\mathrm{CH}$ (Fig. 1a) excludes punctuation marks and words outside of any chunk.

Constituency tree representation (CONST). Standard constituency tree representation.

Dependency tree representations. We provide three dependency-based relational structures: DT1, DT2 and LCT. DT1 (Fig. 1b) is a dependency tree in which grammatical relations become nodes and lemmas are located at the leaf level. DT2 (Fig. 1c) is DT1 modified to include the chunking information, and lemmas in the same chunk are grouped under the same chunk node. Finally, LCT (Fig. 1d) is a lexical-centered dependency tree (Croce et al., 2011) with the grammatical relation $R E L($ head, child) represented as (head (child GR-REL POS-pos(head)). Here REL is a grammatical relation, head and child are the head and child lemmas in the relation, respectively, and pos (head) is the POS-tag of the head lemma. GR- and POS- tags in the node name indicate that the node is a grammar relation or partof-speech node, respectively.

\subsection{RelTextRank relational links}

Experimental results on multiple datasets (Severyn et al., 2013; Severyn and Moschitti, 2012) show that encoding information about the relations between $T_{1}$ and $T_{2}$ is crucial for obtaining the state of the art in text pair ranking and classification. RelTextRank provides two kinds of links: hard string match, REL, and semantic match, FREL.

REL. If some lemma occurs both in $T_{1}$ and $T_{2}$, we mark the respective POS-tag nodes and their parents in the structural representations of $T_{1}$ and $T_{2}$ with REL labels for all the structures, except for LCT. In LCT, we mark with REL- the POS (POS-) and grammar relation (GR-) nodes.

FREL. When working in the question answering setting, i.e., when $T_{1}$ is a question and $T_{2}$ is a candidate answer passage, we encode the question focus and question class information into the structural representations. We use $\mathrm{EREL}-\langle\mathrm{QC}>$ tag to mark question focus in $T_{1}$. Then, in $T_{2}$, we mark all the named entities $T_{2}$ of type compatible with the question class $^{3}$. Here, $\langle Q \mathrm{QC}\rangle$ is substituted

\footnotetext{
${ }^{3}$ We use the following mappings to check for compatibility(Stanford named entity type $\rightarrow$ UIUC question class (Li and Roth, 2002)): Person, Organization $\rightarrow$ HUM ,ENTY; Misc $\rightarrow$ ENTY; Location $\rightarrow$ LOC; Date, Time, Money, Percentage, Set, Duration $\rightarrow$ NUM
} 


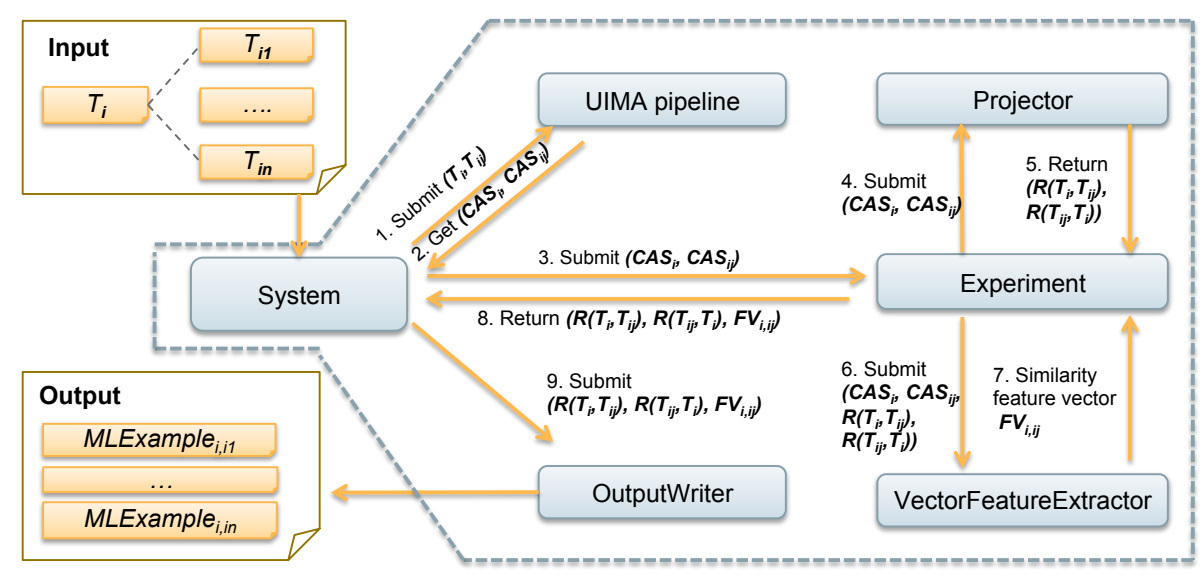

Figure 2: Overall framework

with an actual question class. For all structures in Sec. 2.1, except LCT, we prepend the FREL$\langle Q C>$ tag to the grand-parents of the lemma nodes. In case of LCT, we mark the child grammatical (GR-) and POS-tag (POS-) nodes of the lemma nodes. Finally, only in case of $\mathrm{CH}^{p}$ structure we also add $\angle Q C>$ to the ROOT tree node as its rightmost child, and mark the question focus node simply as FREL. Fig. 1e shows an example of a $\mathrm{CH}^{p}$ enriched with REL and FREL links. ${ }^{4}$

\section{System Architecture}

RelTextRank has a modular architecture easy to adjust towards new structures, features and relational links (see Fig. 2). The basic input of RelTextRank is a text $T_{i}$, and a list of $n$ texts, $T_{i 1}, \ldots, T_{i n}$ to be classified or reranked as relevant or not for $T_{i}$. For example, in the QA setting, $T_{i}$ would be a question and $T_{i j}, j=1, \ldots, n$ would be a list of $n$ candidate answer passages. The output of the system is a file in the SVMLight-TK ${ }^{5}$ format containing the relational structures and feature vectors generated from the $\left\langle T_{i},\left(T_{i 1}, \ldots, T_{i n}\right)\right\rangle$ tuples.

When launched, the RelTextRank System module first initializes the other modules, such as the UIMA text analysis pipeline responsible for linguistic annotation of input texts, the Experiment module responsible for generating the structural representations enriched with the relational labels, and, finally, the OutputWriter module, which generates the output in the SVMLight-TK format.

At runtime, given an input $\left\langle T_{i},\left(T_{i 1}, \ldots, T_{i n}\right)\right\rangle$ tuple, RelTextRank generates $\left(T_{i}, T_{i j}\right)$ pairs $(j=$ $1, \ldots, n)$, and performs the following steps:

\footnotetext{
${ }^{4}$ Note that the structures in Fig. 1a- 1d here are depicted without REL and FREL links, however, at runtime the classes described in Sec. 4 do enrich them with the links

5 http://disi.unitn.it/moschitti/Tree-Kernel.htm
}

Step 1. Linguistic annotation. It runs a pipeline of UIMA Analysis Engines (AEs), which wrap linguistic annotators, e.g., Sentence Splitters, Tokenizers, Syntactic parsers, to convert the input text pairs $\left(T_{i}, T_{i j}\right)$ into the UIMA Common Analysis Structures (CASes), i.e., $\left(C A S_{i}\right.$ and $\left.C A S_{i j}\right)$. CASes contain the original texts and all the linguistic annotations produced by AEs. These produce linguistic annotations defined by a UIMA Type System. In addition, there is an option to persist the produced CASes, and not to rerun the annotators when re-processing a specific document.

Step 2. Generation of structural representations and feature vectors. The Experiment module is the core architectural component of the system, which takes CASes as input and generates the relational structures for $T_{i}$ and $T_{i j}$ along with their feature vector representation, $F V_{i, i j} . R\left(T_{i}, T_{i j}\right)$ is the relational structure for $\mathrm{T}_{i}$ enriched with the relational links towards $T_{i j}$, while $R\left(T_{i j}, T_{i}\right)$ is the opposite, i.e., the relational structure for $T_{i j}$ with respect to $T_{i}$. Here, the Projector module generates $\left(R\left(T_{i}, T_{i j}\right), R\left(T_{i j}, T_{i}\right)\right)$ and the VectorFeatureExtractor module generates $F V_{i, i j}$. In Sec. 4, we provide a list of Experiment modules that implement the representations described in Sec. 2.1, and a list of feature extractors to generate $F V_{i, i j}$.

Step 3. Generation of the output files. Once, all the pairs generated from the $\left\langle T_{i},\left(T_{i 1}, \ldots, T_{i n}\right)\right\rangle$ tuple have been processed, the OutputWriter module writes them into training/test files. We provide several output strategies described in Sec. 4.

\section{Architecture Components}

In order to generate the particular configuration of train/test data, one must specify which System, Experiment and VectorFeatureExtractor modules 


\begin{tabular}{l|l} 
Structure/previous usage & Class Name \\
\hline $\begin{array}{l}\text { CH (Severyn et al., 2013; Tymoshenko et al., } \\
\text { 2016b) }\end{array}$ & CHExperiment \\
\hline $\begin{array}{l}\text { DT1 (Severyn et al., 2013; Tymoshenko and } \\
\text { Moschitti, 2015) }\end{array}$ & DT1Experiment \\
\hline DT2 (Tymoshenko and Moschitti, 2015) & DT2Experiment \\
\hline $\begin{array}{l}\text { LCT }{ }_{Q} \text {-DT2 }{ }_{A} \text { (Tymoshenko and Moschitti, } \\
\text { 2015) }\end{array}$ & LCTqDT2aExperiment \\
\hline CONST & ConstExperiment \\
\hline CH ${ }^{p}$ (Tymoshenko et al., 2016a) & CHpExperiment \\
\hline CH-cQA (Barrón-Cedeño et al., 2016) & CHcQaExperiment \\
\hline CONST-cQA (Tymoshenko et al., 2016b) & ConstQaExperiment
\end{tabular}

Table 1: List of experimental configurations

to be used. In this section, we describe the implementations of the architectural modules currently available within RelTextRank.

System modules. These are the entry point to the pipeline, they initialize the specific structure and feature vector generation strategies (Experiment and VectorFeatureExtractor) and define the type (classification or reranking) and the format of the output file. Currently, we provide the system modules for generating classification (ClassTextPairConversion) and reranking training (RERTextPairConversion) files. Then, we provide a method to generate the cross-validation experimental data (ClassCVTextPairConversion and CVRERTextPairConversion). Additionally, we provide a method for generating training/test data for the answer comment reranking in cQA, CQASemevalTaskA. Finally, we provide a prediction module for classifying and reranking new text pairs with a pre-trained classifier (TextPairPrediction).

Every System module uses a single OutputWriter module, whose type of Experiment and FeatureVectorExtractor to be used are specified with command line parameters (see Sec. 5.)

UIMA pipeline. We provide the UIMA AEs (see it.unitn.nlpir.annotators), wrapping the components of the Stanford pipeline (Manning et al., 2014) and Illinois Chunker (Punyakanok and Roth, 2001). The UIMA pipeline takes a pair of texts, $\left(T_{i}, T_{i j}\right)$ as input, and outputs their respective CASes, $\left(C A S_{i}, C A S_{i j}\right)$.

RelTextRank also includes AEs for question classification (Questionclassifier) and question focus detection (QuestionFocusAnnotator). Focus classification module employs a model pre-trained as in (Severyn et al., 2013). Questionclassifier can be run with coarse- and fine-grained question classification models trained on the UIUC corpus by ( $\mathrm{Li}$ and

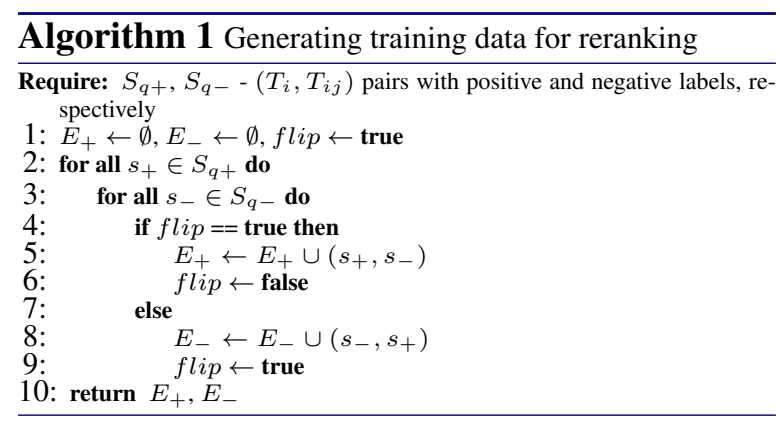

Roth, 2002) as described in (Severyn et al., 2013). The coarse-grained classier uses the following categories, HUMan, ENTitY, LOCation, ABBR, DESCription, NUMber classes, whereas the fine-grained classifier splits the NUM class into DATE, CURRENCY and QUANTITY.

Currently, we use a custom UIMA type system defined for our pipeline, however, in future we plan to use the type systems used in other widely used UIMA pipelines, e.g., DKPro (de Castilho and Gurevych, 2014).

Experiment modules. All the Experiment module implementations are available as classes in the it.unitn.nlpir.experiment.* package. Tab. 1 provides an overview of the structures currently available within the system. Here $\mathrm{LCT}_{Q^{-}}$ DT2 ${ }_{A}$ represents $T_{i}$ and $T_{i j}$ as LCT and DT2 structures, respectively. $\mathrm{CH}-\mathrm{cQA}$ and CONSTcQA are the $\mathrm{CH}$ and CONST structures adjusted for cQA (see (Tymoshenko et al., 2016b)).

OutputWriter modules. In the it.unitn. nlpir.system.datagen package, we provide the OutputWriters, which output the data in the SVMLight-TK format in the classification (ClassifierDataGen) and the reranking (RerankingDataGenTrain and RerankingDataGenTest) modes. Currently, the type of the OutputWriter can only be specified in the code of the System module. It is possible to create a new System module starting from the existing one and code a different OutputWriter.

In the classification mode, one OutputWriter generates one example for each text pair $\left(T_{i}, T_{i j}\right)$. Another OutputWriter implementation generates input data for kernel-based reranking (Shen et al., 2003) using the strategy described in Alg. 1.

VectorFeatureExtractors. RelTextRank contains feature extractors to compute: (i) cosine similarity over the text pair: $\operatorname{sim}_{C O S}\left(T_{1}, T_{2}\right)$, where the input vectors are composed of word lemmas, bi-, three- an four-grams, POS-tags; similarity based on the PTK score computed for the structural rep- 
Command 1. Generate training data

java -Xmx5G -Xss512m it.unitn.nlpir.system.core.RERTextPairConversion -questionsPath data/wikiQA/WikiQA-train.questions.txt -answersPath data/wikiQA/WikiQA-train.tsv.resultset -outputDir data/examples/wikiqa -filePersistence CASes/wikiQA -candidatesToKeep 10 -mode train -expClassName it.unitn.nlpir.experiment.fqa.CHExperiment

-featureExtractorClass it.unitn.nlpir.features.presets.BaselineFeatures

Command 2. Use pre-trained model to do classification

java -Xmx5G -Xss512m it.unitn.nlpir.system.core.TextPairPrediction -svmModel data/wikiQA/wikiqa-ch-rer-baselinefeats.model -featureExtractorClass it.unitn.nlpir.features.presets.BaselineFeatures -questionsPath data/wikiQA/WikiQA-test.questions.txt -answersPath data/wikiQA/WikiQA-test.tsv.resultset -outputFile data/examples/wikiqa/wikiqa-ch-rer-baselinefeats.pred -expClassName it.unitn.nlpir.experiment.fqa.CHExperiment -mode reranking -filePersistence CASes/wikiQA/test

Table 2: Example commands to launch the pipeline

resentations of $T_{1}$ and $T_{2}: \operatorname{sim}_{P T K}\left(T_{1}, T_{2}\right)=$ $P T K\left(T_{1}, T_{2}\right)$, where the input trees can both be the dependency trees and/or the shallow chunk trees; (ii) IR score, which is a normalized score assigned to the answer passage by an IR engine, if available; (iii) question class as a binary feature.

Then, RelTextRank includes feature extractors based on the DKPro Similarity tool (Bär et al., 2013) for extracting (iv) longest common substring/subsequence measure; (v) Jaccard similarity coefficient on 1,2,3,4-grams; (vi) word containment measure (Broder, 1997); (vii) greedy string tiling (Wise, 1996); and (viii) ESA similarity based on Explicit Semantic Analysis (ESA) (Gabrilovich and Markovitch, 2007),

We provide several predefined VectorFeatureExtractors: BaselineFeatures, AllFeatures and NoESAAllFeatures ${ }^{6}$, which incorporate feature groups: (i)-(iii), (i)-(viii) and (i)-(vii), respectively. Finally, we provide a feature extractor that reads the pre-extracted features from file, FromfileVectorFeature. The full list of features can be found by exploring the contents and documentation of the it.unitn.nlpir.features package.

\section{Running the pipeline}

Documentation on the RelTextRank GitHub explains how to install and run the pipeline with the various reranking and classification configurations. Due to the space limitations, here we only provide the sample commands for running the training file generation for the reranking mode (Tab. 2, Command 1) and using a pretrained model to rank the produced data (Tab. 2, Command 2).

Command 1 runs the RERTextPairConversion system (see Sec. 4), using the files specified as -questionsPath and -answersPath parameters to read the questions ( $T_{i}$ in Fig 2 ) and their corre-

\footnotetext{
${ }^{6}$ The motivation behind this feature extractor is that ESA feature extraction process is time-consuming
}

sponding candidate answers $\left(T_{i j}\right.$, in Fig 2 with $j=1, \ldots, n$; -candidatesToKeep parameter specifies the value of $n$ ), respectively. -outputDir is a path to the folder that will contain the resulting training file, while -filePersistence indicates where to persist the UIMA CASes containing the linguistic annotations produced by the UIMA pipeline (this is optional). -mode train indicates that we are generating the training file. -expClassName is a mandatory parameter, which indicates the Experiment module (Fig. 2) we want to invoke, i.e., which structure we wish to generate. In this specific example, we build a $\mathrm{CH}$ structure (see Tab. 1). Finally, -featureExtractorClass specifies which features to include into the feature vector.

Command 2 runs a pipeline that uses a pretrained SVMLight-TK model (-svmModel parameter) to rerank the candidate answers (-answerPath) for the input questions (-questionsPath), and stores them into a prediction file (-outputFile). Here, we also indicate which structure generator and feature extractors to be used (-expClassName and featureExtractorClass). Note that -expClassName and -featureExtractorClass must be exactly the same as the ones used when generating the data for training the model specified by svmModel.

\section{Previous uses of RelTextRank}

Tab. 3 reports the results of some of the stateof-the-art AS and cQA systems that employ Re 1TextRank as a component and combine the structures produced by it with the feature vectors of different nature, $V$. Here feature vectors are either manually handcrafted thread-level features, $V_{t}$, or word and phrase vector features, $V_{b}$, for cQA; or embeddings of $T_{i}, T_{i j}$ learned by Convolutional Neural Networks, $V_{C N N}$, for the AS task.

Due to space limitations, we do not describe every system in detail, but provide link to a reference paper with the detailed setup description, and 


\begin{tabular}{|c|c|c|c|c|c|c|c|}
\hline \multirow{2}{*}{ Corpus } & \multirow{2}{*}{ Reference paper } & \multirow{2}{*}{ Struct. } & \multirow{2}{*}{ Feat. } & \multicolumn{2}{|c|}{ V } & \multicolumn{2}{|c|}{ + Rel.Structures } \\
\hline & & & & MRR & MAP & MRR & MAP \\
\hline WikiQA & (Tymoshenko et al., 2016a) $^{*}$ & $\mathrm{CHp}$ & $\mathrm{V}_{C N N}$ & 67.49 & 66.41 & 73.88 & 71.99 \\
\hline TREC13 & (Tymoshenko et al., 2016a) & $\mathrm{CH}$ & $\mathrm{V}_{C N N}$ & 79.32 & 73.37 & $85.53 *$ & $75.18^{*}$ \\
\hline SemEval-2016, 3.A, English & (Tymoshenko et al., 2016b) & CONST & $\mathrm{V}_{t}$ & 82.98 & 73.50 & 86.26 & 78.78 \\
\hline SemEval-2016, 3.D, Arabic & (Barrón-Cedeño et al., 2016) & CONST & $\mathrm{V}_{b}$ & 43.75 & 38.33 & 52.55 & 45.50 \\
\hline
\end{tabular}

Table 3: Previous uses of RelTextRank

mention which of the structures described in Sec. 2 they employ. (Tymoshenko et al., 2016a)* is a new structure and embedding combination approach.

We show the results on two AS corpora, WikiQA (Yang et al., 2015) and TREC13 (Wang et al., 2007). Then, we report the results obtained when using RelTextRank in a cQA system for English and Arabic comment selection tasks in the SemEval-2016 competition, Tasks 3.A and 3.D (Nakov et al., 2016).

$V$ column reports the performance of the systems that employ feature vectors only, while + Rel.Structures corresponds to the systems using a combination of relational structures generated by the earlier versions of Rel TextRank and feature vectors. The numbers marked by $*$ were obtained using relational structures only, since combining features and trees decreased the overall performance in that specific case. Rel.Structures always improves the performance.

\section{Conclusions}

In this demonstration paper we have provided an overview of the architecture and the particular components of the RelTextRank pipeline for generating structural relational representations of text pairs. In previous work, these representations have shown to achieve the state of the art for factoid QA and cQA. In the future, we plan to further evolve the pipeline, improving its code and usability. Moreover, we plan to expand the publicly available code to include more relational links, e.g., Linked Open Data-based relations described in (Tymoshenko et al., 2014). Finally, in order to enable better compatibility with publicly available tools, we plan to adopt the DKPro type system (de Castilho and Gurevych, 2014).

\section{Acknowledgments}

This work has been partially supported by the EC project CogNet, 671625 (H2020-ICT-2014-2, Research and Innovation action).

\section{References}

D. Bär, T. Zesch, and I. Gurevych. 2013. Dkpro similarity: An open source framework for text similarity. In ACL: System Demonstrations.
A. Barrón-Cedeño, G. Da San Martino, S. Joty, A. Moschitti, F. Al-Obaidli, S. Romeo, K. Tymoshenko, and A. Uva. 2016. Convkn at semeval-2016 task 3: Answer and question selection for question answering on arabic and english fora. In SemEval-2016.

A. Z Broder. 1997. On the resemblance and containment of documents. In SEQUENCES.

D. Croce, A. Moschitti, and R. Basili. 2011. Structured lexical similarity via convolution kernels on dependency trees. In $E M N L P$.

R. Eckart de Castilho and I. Gurevych. 2014. A broadcoverage collection of portable NLP components for building shareable analysis pipelines. In OIAF4HLT Workshop (COLING).

E. Gabrilovich and S. Markovitch. 2007. Computing semantic relatedness using wikipedia-based explicit semantic analysis. In IJCAI.

X. Li and D. Roth. 2002. Learning question classifiers. In COLING.

C. D. Manning, M. Surdeanu, J. Bauer, J. Finkel, S. J. Bethard, and D. McClosky. 2014. The Stanford CoreNLP natural language processing toolkit. In $A C L$.

P. Nakov, L. Màrquez, A. Moschitti, W. Magdy, H. Mubarak, A. Freihat, J. Glass, and B. Randeree. 2016. Semeval2016 task 3: Community question answering. In SemEval.

V. Punyakanok and D. Roth. 2001. The use of classifiers in sequential inference. In NIPS.

A. Severyn and A. Moschitti. 2012. Structural relationships for large-scale learning of answer re-ranking. In SIGIR.

A. Severyn, M. Nicosia, and A. Moschitti. 2013. Learning adaptable patterns for passage reranking. In CoNLL.

L. Shen, A. Sarkar, and A. Joshi. 2003. Using LTAG Based Features in Parse Reranking. In EMNLP.

K. Tymoshenko, D. Bonadiman, and A. Moschitti. 2016a. Convolutional neural networks vs. convolution kernels: Feature engineering for answer sentence reranking. In NAACL-HLT .

K. Tymoshenko, D. Bonadiman, and A. Moschitti. 2016b. Learning to rank non-factoid answers: Comment selection in web forums. In CIKM.

K. Tymoshenko and A. Moschitti. 2015. Assessing the impact of syntactic and semantic structures for answer passages reranking. In $C I K M$.

K. Tymoshenko, A. Moschitti, and A. Severyn. 2014. Encoding semantic resources in syntactic structures for passage reranking. In $E A C L$.

Mengqiu Wang, Noah A. Smith, and Teruko Mitaura. 2007. What is the Jeopardy model? A quasi-synchronous grammar for QA. In EMNLP-CoNLL.

Michael J. Wise. 1996. Yap3: improved detection of similarities in computer program and other texts. In ACM SIGCSE Bulletin.

Y. Yang, W. Yih, and C. Meek. 2015. Wikiqa: A challenge dataset for open-domain question answering. In EMNLP. 